\title{
Numerical simulation of acoustic time reversal mirrors
}

\author{
Chokri Ben Amar* Nabil Gmati* Christophe Hazard ${ }^{\dagger}$ \\ Karim Ramdani ${ }^{\ddagger}$
}

February 24, 2013

\begin{abstract}
We study the time reversal phenomenon in a homogeneous and non-dissipative medium containing sound-hard obstacles. We propose two mathematical models of time reversal mirrors in the frequency domain. The first one takes into account the interactions between the mirror and the obstacles. The second one provides an approximation of these interactions. We prove, in both cases, that the time reversal operator $T$ is selfadjoint and compact. The D.O.R.T method (french acronym for Decomposition of the Time Reversal Operator) is explored numerically. In particular, we show that selective focusing, which is known to occur for small and distant enough scatterers, holds when the wavelength and the size of these scatterers are of the same order of magnitude (medium frequency situation). Moreover, we present the behaviour of the eigenvalues of $T$ according to the frequency and we show their oscillations due to the interactions between the mirror and the obstacles and between the obstacles themselves.
\end{abstract}

Keywords: Time reversal, frequency domain, acoustic scattering, selective focusing

AMS: 31B10, 35P25, 74J20

*LAMSIN, ENIT, Campus universitaire B.P. 37, 1002 Tunis Belvédère, Tunisie (chokri.benamar@lamsin.rnu.tn and nabil.gmati@ipein.rnu.tn).

†POEMS (CNRS / ENSTA / INRIA), 32, Boulevard Victor, 75739 Paris Cedex 15, France (christophe.hazard@ensta.fr).

†INRIA (Projet CORIDA) et Institut Elie Cartan de Nancy, Université de Nancy I, Vandoeuvre-les-Nancy, 54506, France (karim.ramdani@loria.fr). 


\section{Introduction}

During the last decade, time reversal techniques have been extensively studied, in particular for detection, localization and identification of scatterers in propagative media. In the present paper, we are concerned with one of these techniques, usually referred to as the D.O.R.T method (french acronym for Decomposition of the Time Reversal Operator). This method was first developed by Prada and Fink [17] in the context of ultrasonics (see [18] for an overview). It consists in determining the invariants of a time reversal process which can be described as follows. A time reversal mirror (TRM), composed of an array of transducers, first emits an incident wave corresponding to a given distribution of signals sent to the transducers. This wave is then scattered by the presence of obstacles in the propagative medium. In a second step, the TRM measures the scattered field and time-reverses the measure, which furnishes a new distribution of signals used to re-emit a new incident wave. In short, one cycle of the process corresponds to the succession: emission, scattering, measure, time reversal. The so-called time reversal operator $T$ is obtained by iterating this cycle twice. The D.O.R.T method deals with the eigenvalues of $T$ and the associated eigenvectors for a fixed frequency, that is when time-harmonic waves are considered. In this case, time reversal simply amounts to a phase conjugation. It was shown [17, 19] and confirmed by experiments that for ideally resolved or pointlike and distant enough scatterers with different reflectivities, each eigenvector corresponding to a nonzero eigenvalue of $T$ provides the signals to be sent to the transducers in order to focus on one scatterer. A mathematical justification of these selective focusing properties is given in [8] for a far field approach, i.e., for an ideal TRM which reverses the asymptotic behaviour at large distance of the wave scattered by the obsatcles (in this case, the time reversal operator is related with the far field operator [13] well known in scattering theory). Other applications of the D.O.R.T. method, which concern this question of focusing on a selected target, have been developed: acoustic waveguides [10, 15], electromagnetic scattering [22, 14] or propagation in random media [4].

The focusing properties of the eigenvectors of the time reversal operator are known to occur for small enough scatterers, i.e., when the diameters of the scatterers are small compared to the wavelength. Such a situation corresponds to a low frequency case. The object of the present paper is to explore the medium frequency case by a numerical approach, i.e., when the diameters and the wavelength have the same order of magnitude. The model 
considered here differs from commonly used models in the fact that the TRM is intrusive: instead of an array of pointlike transducers, the TRM consists of a volumic and non penetrable object which perturbs the acoustic field. For the sake of simplicity, we consider the usual simplified model of linear electroacoustic transducers (see, e.g. [16]) : the inner behaviour of the TRM is modelled by a Robin condition on its boundary.

The paper is organized as follows. In 92, we present a mathematical model of a non penetrable intrusive TRM, which is closely related to the active sonar problem dealt with for instance in [20]. In this first model, the interactions between the scatterers and the TRM are taken into account, so that we can deal with the case where they are close to each other. Instead of the symmetric matrix obtained for a finite number of pointlike transducers, the time reversal operator appears then, like in the far field approach [8], as an operator acting in a $L^{2}$ space representing the finite energy space of possible excitations. The basic properties of this operator, namely selfadjointness and compactness, are proved in $\$ 3$. They essentially tell us that its spectrum is that of a symmetric matrix completed by an infinite number of a non significant eigenvalues. In \&4, we propose a non penetrable intrusive model of TRM in which the interactions between the obstacles and the TRM are approximated. We briefly show how to adapt the proofs of 93 . Finally we present some numerical results in \$5. We show that the expected selective focusing properties hold in the medium frequency case. Moreover, we point out the modulations of the eigenvalues of $T$ with respect to the frequency. These oscillations are due to the interactions between the scatterers and the TRM, and between the scatterers themselves.

The main result of this paper, namely the properties of the time reversal operator (Theorem 1), holds in many other situations which can be dealt with by similar integral techniques. For instance, we consider here sound-hard obstacles but we could have chosen a Dirichlet or Robin boundary condition on $\partial \mathcal{O}$ instead of the Neumann condition. Penetrable scatterers, i.e., inhomogeneities of the medium, can also be considered.

\section{A model of non penetrable intrusive mirror}

We consider a homogeneous medium filling the space $\mathbb{R}^{n}(n=2$ or 3 ) and containing a non penetrable mirror $M$ and some non penetrable obstacles $\mathcal{O}$.

We study the case of an impedance condition on the boundary $\partial M$ of 
the mirror and a Neumann condition on the boundary $\partial \mathcal{O}$ of the obstacles (sound-hard obstacles). Let $\Omega_{M}=\mathbb{R}^{n} \backslash \bar{M}, \Omega_{\mathcal{O}}=\mathbb{R}^{n} \backslash \overline{\mathcal{O}}$ and $\Omega_{M, \mathcal{O}}=$ $\mathbb{R}^{n} \backslash(\bar{M} \cup \overline{\mathcal{O}})$. We suppose that the boundary of the mirror is excited by a signal $g$ (proportional to the current which flows through each transducer). So, in the presence of the obstacles, we observe the total field $\varphi_{T}$ satisfying the problem

$$
\left\{\begin{aligned}
\Delta \varphi_{T}+k^{2} \varphi_{T} & =0 \text { in } \Omega_{M, \mathcal{O}} \\
\frac{\partial \varphi_{T}}{\partial n}+\mu \varphi_{T} & =g \text { on } \partial M \\
\frac{\partial \varphi_{T}}{\partial n} & =0 \text { on } \partial \mathcal{O} \\
\text { R.C. at } \infty &
\end{aligned}\right.
$$

where $n$ denotes the unit normal vector directed into the interior of the domain $\Omega_{M, \mathcal{O}}$. The wave number $k$ is defined by $k=\omega / c$ where $\omega$ is the frequency and $c$ is the speed of sound in the homogeneous medium, $\mu$ is a real parameter which represents the inverse of the open-circuit acoustic impedance of the TRM [16] and R.C. the outgoing Sommerfeld's "Radiation Condition" which, for $\varphi_{T}$ is

$$
\lim _{r \rightarrow+\infty} r^{\frac{n-1}{2}}\left(\frac{\partial \varphi_{T}}{\partial r}(x)-i k \varphi_{T}(x)\right)=0, \quad r=|x|,
$$

where $\partial \varphi_{T} / \partial r$ denotes the radial derivative of $\varphi_{T}$.

In the absence of obstacles, we should observe an incident field $\varphi_{I}$ solution to

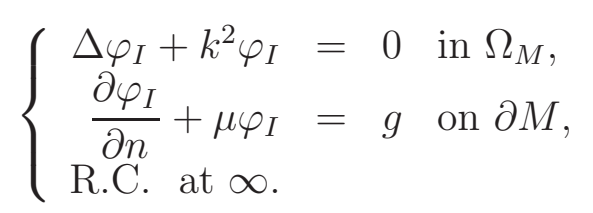

The perturbation due to the presence of the obstacles is the diffracted field $\varphi_{D}=\varphi_{T}-\varphi_{I}$ satisfying the problem

$$
\left\{\begin{aligned}
\Delta \varphi_{D}+k^{2} \varphi_{D} & =0 \text { in } \Omega_{M, \mathcal{O}} \\
\frac{\partial \varphi_{D}}{\partial n}+\mu \varphi_{D} & =0 \text { on } \partial M \\
\frac{\partial \varphi_{D}}{\partial n} & =h \text { on } \partial \mathcal{O} \\
\text { R.C. at } \infty &
\end{aligned}\right.
$$

where $h=-\partial \varphi_{I} / \partial n$. 
We suppose that the signal measured by the mirror is equal to $\varphi_{D_{/ \partial M}}$, the value of the diffracted field on $\partial M$. The measured signal is then conjugated and used to generate the incident and the total fields in the next iteration.

\section{Time reversal operator}

Let $R$ denote the operator describing the response of the medium, that is, the three successive steps: emission, diffraction, measure. It is defined by

$$
R g=\varphi_{D / \partial M}
$$

The time reversal operator is obtained by iterating the time reversal process (emission, diffraction, measure, conjugation) twice. Therefore, $T$ is given by

$$
T g=\overline{R \overline{R g}} \text {, that is, } T=\bar{R} R,
$$

where the operator $\bar{R}$ is defined by

$$
\bar{R} g=\overline{R \bar{g}}
$$

Theorem 1. $T$ is a selfadjoint positive and compact operator in $L^{2}(\partial M)$.

These properties are proved below by an integral approach based on the use of several Green's functions.

\section{Proof of Theorem 1}

This section is devoted to the proof of the following result, from which Theorem 1 derives.

Proposition 2. For every $g \in L^{2}(\partial M)$, the response $R g \in L^{2}(\partial M)$ of the medium is given by

$$
(R g)(x)=\int_{\partial M} G_{R}(x, y) g(y) d \sigma(y),
$$

where $G_{R} \in L^{2}(\partial M \times \partial M)$ is symmetric, i.e., $G_{R}(x, y)=G_{R}(y, x)$. 
This proposition shows that $R$ is a Hilbert-Schmidt operator in $L^{2}(\partial M)$ such that $R^{*}=\bar{R}$ since

$$
\left(R^{*} g\right)(x)=\int_{\partial M} \overline{G_{R}(y, x)} g(y) d \sigma(y) \text { and }(\bar{R} g)(x)=\int_{\partial M} \overline{G_{R}(x, y)} g(y) d \sigma(y) .
$$

Hence $T=R^{*} R$ is selfadjoint positive and compact in $L^{2}(\partial M)$. It is actually a Hilbert-Schmidt operator in $L^{2}(\partial M)$ whose kernel $G \in L^{2}(\partial M \times \partial M)$ is given by

$$
G(x, y)=\int_{\partial M} \overline{G_{R}(z, x)} G_{R}(z, y) d \sigma(z) .
$$

The spectral properties of $T$ follow. On one hand, the eigenvalues of $T$ form a decreasing sequence of positive numbers $\left(\lambda_{n}\right)_{n \in \mathbb{N}^{*}}$ such that $\sum_{n \in \mathbb{N}^{*}} \lambda_{n}^{2}$ is finite. On the other hand, one can choose an orthonormal basis of $L^{2}(\partial M)$ composed of eigenvectors of $T$, and $T$ becomes diagonal in this basis.

\section{Integral representations}

To prove Proposition 2, first recall that problems (2.1), (2.3) and (2.4) are well-posed [7] in a proper functional framework which is made precise later. Consider then the operators

$$
\begin{array}{llllll}
S_{T}: & g & \mapsto \varphi_{T} & \text { solution to (2.1), } \\
S_{I}: & : & g & \mapsto \varphi_{I} & \text { solution to (2.3) }, \\
S_{D}: & h \mapsto \varphi_{D} & \text { solution to (2.4), }
\end{array}
$$

as well as the Green's functions $G_{T}, G_{I}$ and $G_{D}$ which are respectively outgoing solutions (in the sense that they satisfy the outgoing radiation condition (2.21) ) to

$$
\begin{aligned}
& \left\{\begin{array}{rll}
\Delta G_{T}(x, .)+k^{2} G_{T}(x, .) & =\delta_{x} & \text { in } \Omega_{M, \mathcal{O}} \\
\Theta_{M} G_{T}(x, .) & =0 & \text { on } \partial M \\
\Theta_{\mathcal{O}} G_{T}(x, .) & =0 & \text { on } \partial \mathcal{O}
\end{array}\right. \\
& \left\{\begin{aligned}
\Delta G_{I}(x, .)+k^{2} G_{I}(x, .) & =\delta_{x} \text { in } \Omega_{M}, \\
\Theta_{M} G_{I}(x, .) & =0 \text { on } \partial M,
\end{aligned}\right. \\
& \left\{\begin{aligned}
\Delta G_{D}(x, .)+k^{2} G_{D}(x, .) & =0 & & \text { in } \Omega_{M, \mathcal{O}} \\
\Theta_{M} G_{D}(x, .) & =0 & & \text { on } \partial M \\
\Theta_{\mathcal{O}} G_{D}(x, .) & =-\Theta_{\mathcal{O}} G_{I}(x, .) & & \text { on } \partial \mathcal{O},
\end{aligned}\right.
\end{aligned}
$$


where $\delta_{x}$ stands for the Dirac measure at point $x, \Theta_{M}=(\partial / \partial n+\mu) / \partial M$ and $\Theta_{\mathcal{O}}=(\partial / \partial n)_{/ \partial \mathcal{O}}$. By construction, we have $G_{T}=G_{I}+G_{D}$.

These functions can be expressed by means of the usual Green's function $G_{0}$ of the Helmholtz operator in the free space, i.e., the outgoing solution in $\mathbb{R}^{n}$ to $\Delta G_{0}(x,)+.k^{2} G_{0}(x,)=.\delta_{x}$, which is given by

$$
G_{0}(x, y)= \begin{cases}-\frac{e^{i k|x-y|}}{4 \pi|x-y|} & \text { if } n=3 \\ \frac{1}{4 i} H_{0}^{(1)}(k|x-y|) & \text { if } n=2 .\end{cases}
$$

Indeed, we have

$$
\begin{aligned}
& G_{I}(x, .)=G_{0}(x, .)+\widetilde{G}_{I}(x, .) \text { where } \widetilde{G}_{I}(x, .)=-S_{I} \Theta_{M} G_{0}(x, .), \\
& G_{D}(x, .)=-S_{D} \Theta_{\mathcal{O}} G_{I}(x, .)
\end{aligned}
$$

Lemma 3. Let $\Omega_{i}$ stand for $\Omega_{M}$ if $i=I$, and for $\Omega_{M, \mathcal{O}}$ if $i=T$ or $D$. Then $\varphi_{T}=S_{T} g, \varphi_{I}=S_{I} g$ and $\varphi_{D}=-S_{D} \Theta_{\mathcal{O}} \varphi_{I}$ are given by

$$
\varphi_{i}(x)=\int_{\partial M} G_{i}(x, y) g(y) d \sigma(y) \quad \forall x \in \Omega_{i}, i \in\{T, I, D\},
$$

where the kernels $G_{i}$ are symmetric: $G_{i}(x, y)=G_{i}(y, x)$.

Proof. Formulas (3.6) are classical. For the sake of clarity, we recall briefly how to derive them from the usual integral representation [5]

$$
\varphi_{i}(x)=\int_{\partial \Omega_{i}}\left\{G_{0}(x, y) \frac{\partial \varphi_{i}}{\partial n}(y)-\frac{\partial G_{0}}{\partial n_{y}}(x, y) \varphi_{i}(y)\right\} d \sigma(y) \quad \forall x \in \Omega_{i} .
$$

We use the fact that if two functions $\varphi$ and $\psi$ satisfy the Helmholtz equation either inside a bounded domain $\Lambda$, or outside $\Lambda$ together with the radiation condition (2.2), then we have the reciprocity relation [5]

$$
\int_{\partial \Lambda}\left\{\psi \frac{\partial \varphi}{\partial n}-\frac{\partial \psi}{\partial n} \varphi\right\} d \sigma=0
$$

where the normal derivative can obviously be replaced by $(\partial / \partial n+\mu)$.

For $\varphi_{I}$, we replace $G_{0}$ in (3.7) by $G_{I}-\widetilde{G}_{I}$, which yields two similar integral terms on $\partial M$. Thanks to (3.8), the term involving $\widetilde{G}_{I}$ vanishes. The 
other one reduces to the single layer potential (3.6) by virtue of the boundary conditions satisfied by $\varphi_{I}$ and $G_{I}(x,$.$) (see (2.3) and (3.3) ).$

For $\varphi_{T}$, the same idea applies. The integral terms are now set on $\partial M \cup \partial \mathcal{O}$. The term which involves $\widetilde{G}_{T}=G_{T}-G_{0}$ again vanishes by (3.8). Split the other one, which involves $G_{T}$, into two integrals respectively on $\partial M$ and $\partial \mathcal{O}$. Thanks to the boundary conditions in (2.1) and (3.2), the former simplifies as above to (3.6) whereas the latter vanishes.

Finally, substracting the previous representations yields (3.6) for $\varphi_{D}=$ $\varphi_{T}-\varphi_{I}$.

The symmetry of $G_{I}$ is easily deduced from that of $G_{0}$ by proving that the perturbation term $\widetilde{G}_{I}$ is also symmetric. The integral representation (3.7) of $\widetilde{G}_{I}(x,$.$) reads$

$$
\widetilde{G}_{I}(x, y)=\int_{\partial M}\left\{\Theta_{M} G_{0}(y, z) \widetilde{G}_{I}(x, z)-G_{0}(y, z) \Theta_{M} \widetilde{G}_{I}(x, z)\right\} d \sigma(z),
$$

where the operator $\Theta_{M}$ is understood with respect to $z$. The boundary conditions satisfied by $\widetilde{G}_{I}(x,$.$) and \widetilde{G}_{I}(y,$.$) then yields$

$$
\widetilde{G}_{I}(x, y)=-\int_{\partial M} \Theta_{M} \widetilde{G}_{I}(y, z) \widetilde{G}_{I}(x, z) d \sigma(z)+\int_{\partial M} G_{0}(y, z) \Theta_{M} G_{0}(x, z) d \sigma(z)
$$

Thanks to the reciprocity relation (3.8) applied in $\Omega_{M}$ for the first integral, and in $M$ for the second one, we see that both integrals are symmetric for $(x, y) \in \Omega_{M} \times \Omega_{M}$, hence so is $\widetilde{G}_{I}$.

The symmetry of $G_{D}$ is proved similarly, and that of $G_{T}$ follows.

\section{Functional details}

It is now clear that (3.1) follows from the integral representation (3.6) of $\varphi_{D}$ simply by taking its restriction on $\partial M$ :

$$
G_{R}(x, y)=G_{D}(x, y) \text { for }(x, y) \in \partial M \times \partial M .
$$

Hence Proposition 2 will be proved if we are able to justify that this double restriction actually yields a function of $L^{2}(\partial M \times \partial M)$. We thus have to precise the function spaces in which the kernels $G_{i}$ are defined: the appropriate tool to do so is the notion of tensor product of Hilbert spaces [1]. 
All the domains considered are assumed to have Lipschitz boundaries (for instance $\partial M$ and $\partial \mathcal{O}$ may be piece-wise smooth). For a bounded domain $\Lambda \subset \mathbb{R}^{n}$, we denote

$$
\mathbf{H}(\Lambda)=\left\{\varphi \in H^{1}(\Lambda) ; \Delta \varphi \in L^{2}(\Lambda)\right\} .
$$

Recall that, on one hand, the trace operator $\gamma_{\partial \Lambda} \varphi=\varphi / \partial \Lambda$ is continuous from $\mathbf{H}(\Lambda)$ to $H^{1 / 2}(\partial \Lambda)$, and on the other hand, the normal derivative $(\partial \varphi / \partial n)_{/ \partial \Lambda}$ is continuous from $\mathbf{H}(\Lambda)$ to $H^{-1 / 2}(\partial \Lambda)$. Moreover, for every bounded sets $\Lambda_{M} \subset \Omega_{M}$ and $\Lambda_{\mathcal{O}} \subset \Omega_{M, \mathcal{O}}$, the operators $S_{I}$ and $S_{D}$ are continuous from $H^{-1 / 2}(\partial M)$ to $\mathbf{H}\left(\Lambda_{M}\right)$ and from $H^{-1 / 2}(\partial \mathcal{O})$ to $\mathbf{H}\left(\Lambda_{\mathcal{O}}\right)$ (see [7]).

Lemma 4. Let $\Lambda_{M} \subset \Omega_{M}$ and $\Lambda_{\mathcal{O}} \subset \Omega_{M, \mathcal{O}}$ be two bounded sets such that $\partial M \subset \partial \Lambda_{M}, \partial \mathcal{O} \subset \partial \Lambda_{\mathcal{O}}$ and $\overline{\Lambda_{M}} \cap \overline{\Lambda_{\mathcal{O}}}=\emptyset$. Then

$$
G_{I} \in \mathbf{H}\left(\Lambda_{M}\right) \widehat{\otimes} \mathbf{H}\left(\Lambda_{\mathcal{O}}\right) \text { and } G_{D} \in \mathbf{H}\left(\Lambda_{M}\right) \widehat{\otimes} \mathbf{H}\left(\Lambda_{M}\right) .
$$

Proof. Formulas (3.5), which involve operators acting on the second variable $y$, can be rewritten in terms of tensor products of operators as

$$
\begin{aligned}
& G_{I}=G_{0}-\left(I d \otimes S_{I} \Theta_{M}\right) G_{0}, \\
& G_{D}=-\left(I d \otimes S_{D} \Theta_{\mathcal{O}}\right) G_{I} .
\end{aligned}
$$

Let us first deal with $G_{I}$. Thanks to its symmetry, the announced property amounts to showing that $G_{I} \in \mathbf{H}\left(\Lambda_{\mathcal{O}}\right) \widehat{\otimes} \mathbf{H}\left(\Lambda_{M}\right)$. This clearly holds for $G_{0}$ since it is infinitely differentiable outside the diagonal $x=y$. Moreover the above mentioned properties of $S_{I}$ and traces show that $S_{I} \Theta_{M}$ is continuous from $\mathbf{H}\left(\Lambda_{M}\right)$ to $\mathbf{H}\left(\Lambda_{M}\right)$. As a consequence [1], $I d \otimes S_{I} \Theta_{M}$ is continuous from $\mathbf{H}\left(\Lambda_{\mathcal{O}}\right) \widehat{\otimes} \mathbf{H}\left(\Lambda_{M}\right)$ to itself. The conclusion follows.

For $G_{D}$, we use the previous result and the fact that $I d \otimes S_{D} \Theta_{\mathcal{O}}$ is continuous from $\mathbf{H}\left(\Lambda_{M}\right) \widehat{\otimes} \mathbf{H}\left(\Lambda_{\mathcal{O}}\right)$ to $\mathbf{H}\left(\Lambda_{M}\right) \widehat{\otimes} \mathbf{H}\left(\Lambda_{M}\right)$.

We finally have to notice that since $\gamma_{\partial M}$ is continuous from $\mathbf{H}\left(\Lambda_{M}\right)$ to $L^{2}(\partial M) \supset H^{1 / 2}(\partial M)$, the "double trace" $\gamma_{\partial M} \otimes \gamma_{\partial M}$ is continuous from $\mathbf{H}\left(\Lambda_{M}\right) \widehat{\otimes} \mathbf{H}\left(\Lambda_{M}\right)$ to $L^{2}(\partial M) \widehat{\otimes} L^{2}(\partial M)=L^{2}(\partial M \times \partial M)$. Hence the above lemma yields

$$
G_{R}=\left(\gamma_{\partial M} \otimes \gamma_{\partial M}\right) G_{D} \in L^{2}(\partial M \times \partial M)
$$

which is obviously symmetric. This completes the proof of Proposition 2 , and thus of Theorem 1, 


\section{An approximate model}

The model we consider in this section is an approximation of the model introduced in $\oint_{2}$. Although more intricate in its presentation, it leads to a reduction of the computational cost of the time reversal operator for it separates the respective roles of the TRM and the scatterers. It can be seen as the first steps of an iterative method used in the context of multiple scattering problems (see, e.g., [6, 21] and [3, 2] for a rigorous justification of the method, [12] for an overview). The coupled problem of $\$ 2$ is solved by considering the successive reflections between the TRM and the scatterers. Here only specular waves, i.e., the first reflections, are taken into account. Comparing this model with that of 92 will help us in $\$ 5$ to understand the influence of multiple scattering between the obstacles and the TRM upon the eigenelements of the time reversal operator.

Considering the same incident wave $\varphi_{I}=S_{I} g$ as in 92 , the diffracted field is now approximated near the TRM by a superposition of two waves: $\varphi_{D}=\varphi_{D}^{(1)}+\varphi_{D}^{(2)}$. The first one $\varphi_{D}^{(1)}$ represents the result of the diffraction of $\varphi_{I}$ by the scatterers alone, i.e., the outgoing solution to

$$
\left\{\begin{aligned}
\Delta \varphi_{D}^{(1)}+k^{2} \varphi_{D}^{(1)} & =0 & & \text { in } \Omega_{\mathcal{O}} \\
\Theta_{\mathcal{O}} \varphi_{D}^{(1)} & =-\Theta_{\mathcal{O}} \varphi_{I} & & \text { on } \partial \mathcal{O}
\end{aligned}\right.
$$

The second one is the result of the diffraction of the latter by the TRM alone, i.e., the outgoing solution to

$$
\left\{\begin{aligned}
\Delta \varphi_{D}^{(2)}+k^{2} \varphi_{D}^{(2)} & =0 & & \text { in } \Omega_{M} \\
\Theta_{M} \varphi_{D}^{(2)} & =-\Theta_{M} \varphi_{D}^{(1)} & & \text { on } \partial M
\end{aligned}\right.
$$

We assume again that the TRM measures the trace of $\varphi_{D}$ on $\partial M$. Hence the response of the medium is now described by the operator

$$
R g=\varphi_{D / \partial M}=\left(\varphi_{D}^{(1)}+\varphi_{D}^{(2)}\right) / \partial M .
$$

Theorem 1 holds in this case: the time reversal operator $T=\bar{R} R$ is positive, selfadjoint and compact in $L^{2}(\partial M)$.

The proof is similar to that of section 3, We simply have to replace the Green's function $G_{D}$ by $G_{D}=G_{D}^{(1)}+G_{D}^{(2)}$ where $G_{D}^{(1)}$ and $G_{D}^{(2)}$ are respectively the outgoing solutions to

$$
\left\{\begin{aligned}
\Delta G_{D}^{(1)}(x, .)+k^{2} G_{D}^{(1)}(x, .) & =0 & & \text { in } \Omega_{\mathcal{O}} \\
\Theta_{\mathcal{O}} G_{D}^{(1)}(x, .) & =-\Theta_{\mathcal{O}} G_{I}(x, .) & & \text { on } \partial \mathcal{O}
\end{aligned}\right.
$$


and

$$
\left\{\begin{aligned}
\Delta G_{D}^{(2)}(x, .)+k^{2} G_{D}^{(2)}(x, .) & =0 & & \text { in } \Omega_{M}, \\
\Theta_{M} G_{D}^{(2)}(x, .) & =-\Theta_{M} G_{D}^{(1)}(x, .) & & \text { on } \partial M .
\end{aligned}\right.
$$

Lemma 5. The following integral representation holds:

$$
\forall x \in \Omega_{M, \mathcal{O}}, \varphi_{D}(x)=\int_{\partial M} G_{D}(x, y) g(y) d \sigma(y),
$$

where $G_{D}$ is symmetric in $\Omega_{M, \mathcal{O}} \times \Omega_{M, \mathcal{O}}$.

Proof. Contrary to Lemma 3, we are not able to give an intrinsic definition of the total field $\varphi_{I}+\varphi_{D}$ by means of a problem such as (2.1) which would only depend on the incident field. We thus give a direct proof of the above integral representation, starting from the classical formula (3.7) applied to $\varphi_{D}^{(1)}$ in $\Omega_{\mathcal{O}}$.

Using (3.5) and (3.8) applied to $\widetilde{G}_{I}(x,$.$) and \varphi_{D}^{(1)}$ in $\Omega_{M, \mathcal{O}}$, formula (3.7) becomes

$\forall x \in \Omega_{M, \mathcal{O}}, \varphi_{D}^{(1)}(x)=\int_{\partial M \cup \partial \mathcal{O}}\left\{-\Theta_{\bullet} G_{I}(x, y) \varphi_{D}^{(1)}(y)+G_{I}(x, y) \Theta_{\bullet} \varphi_{D}^{(1)}(y)\right\} d \sigma(y)$.

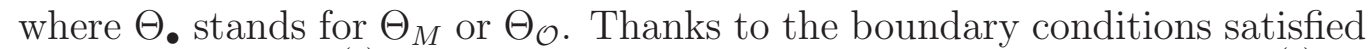
by $G_{I}(x,$.$) and \varphi_{D}^{(2)}$, the contribution on $\partial M$ is nothing but $S_{I} \Theta_{M} \varphi_{D}^{(1)}=$ $-\varphi_{D}^{(2)}$ by Lemma 3 , so the contribution on $\partial \mathcal{O}$ is exactly $\varphi_{D}(x)$. We thus have

$$
\begin{aligned}
\varphi_{D}(x) & =\int_{\partial \mathcal{O}}\left\{\Theta_{\mathcal{O}} G_{D}^{(1)}(x, y) \varphi_{D}^{(1)}(y)-G_{I}(x, y) \Theta_{\mathcal{O}} \varphi_{I}(y)\right\} d \sigma(y) \\
& =\int_{\partial \mathcal{O}}\left\{G_{D}^{(1)}(x, y) \Theta_{\mathcal{O}} \varphi_{D}^{(1)}(y)-\Theta_{\mathcal{O}} G_{I}(x, y) \varphi_{I}(y)\right\} d \sigma(y) \\
& =\int_{\partial \mathcal{O}}\left\{-G_{D}^{(1)}(x, y) \Theta_{\mathcal{O}} \varphi_{I}(y)+\Theta_{\mathcal{O}} G_{D}^{(1)}(x, y) \varphi_{I}(y)\right\} d \sigma(y) \\
& =\int_{\partial M}\left\{G_{D}^{(1)}(x, y) \Theta_{M} \varphi_{I}(y)-\Theta_{M} G_{D}^{(1)}(x, y) \varphi_{I}(y)\right\} d \sigma(y)
\end{aligned}
$$

The first and third equalities result from the boundary conditions satisfied by $G_{D}^{(1)}(x,$.$) and \varphi_{D}^{(1)}$. The second one derives from the reciprocity relation (3.8) applied on one hand to $G_{D}^{(1)}(x,$.$) and \varphi_{D}^{(1)}$ in $\Omega_{\mathcal{O}}$, and on the other hand, to $G_{I}(x,$.$) and \varphi_{I}$ in $\mathcal{O}$. The last one again results from (3.8) 
applied to $G_{D}^{(1)}(x,$.$) and \varphi_{I}$ in $\Omega_{M, \mathcal{O}}$. Noticing finally that $\Theta_{M} G_{D}^{(2)}(x,)=$. $-\Theta_{M} G_{D}^{(1)}(x,$.$) , we have$

$$
\int_{\partial M} \Theta_{M} G_{D}^{(1)}(x, y) \varphi_{I}(y) d \sigma(y)=-\int_{\partial M} G_{D}^{(2)}(x, y) \Theta_{M} \varphi_{I}(y) d \sigma(y),
$$

thanks to (3.8) applied to $G_{D}^{(2)}(x,$.$) and \varphi_{I}$ in $\Omega_{M}$. Since $g=\Theta_{M} \varphi_{I}$, the integral representation of $\varphi_{D}$ follows.

The symmetry of $G_{D}(x,$.$) is proved by the same argument as in Lemma$ 3. Since $G_{D}^{(1)}(x,$.$) and G_{D}^{(2)}(x,$.$) play the same role as \varphi_{D}^{(1)}$ and $\varphi_{D}^{(2)}$, we obtain for $G_{D}(x, y)$ a similar formula as (4.3):

$$
G_{D}(x, y)=\int_{\partial \mathcal{O}}\left\{\Theta_{\mathcal{O}} G_{D}^{(1)}(y, z) G_{D}^{(1)}(x, z)-G_{I}(y, z) \Theta_{\mathcal{O}} G_{I}(x, z)\right\} d \sigma(z) .
$$

where both terms are symmetric by (3.8)

\section{Two-dimensional numerical simulation}

To solve numerically problems (2.3), (2.4), (4.1) and (4.2), we formulate them in bounded domains to apply a finite element method. We use the so-called coupling method between integral representation and finite elements, which is a non singular alternative to the well-known integral equation techniques. This method has been introduced by Jami and Lenoir [9] in hydrodynamics, and then extended to many other wave propagation problems.

\subsection{Bounded domain formulation}

We describe the method only for problem (4.1), but the same technique is also applied for the other ones. We consider a bounded domain $\Omega^{\prime}$ surrounding $\mathcal{O}$ and included in $\Omega_{O}$ (see figure 5.1) and we introduce the following problem set in the domain $\Omega^{\prime}$

$$
\left\{\begin{aligned}
\Delta \varphi^{\prime}+k^{2} \varphi^{\prime} & =0 & \Omega^{\prime} \\
\frac{\partial \varphi^{\prime}}{\partial n} & =h & \partial \mathcal{O} \\
\Theta_{\Sigma} \varphi^{\prime} & =\Theta_{\Sigma} \int_{\partial \mathcal{O}}\left\{G_{0}(., y) \frac{\partial \varphi^{\prime}}{\partial n}(y)-\frac{\partial G_{0}}{\partial n_{y}}(., y) \varphi^{\prime}(y)\right\} d \sigma(y) & \Sigma
\end{aligned}\right.
$$




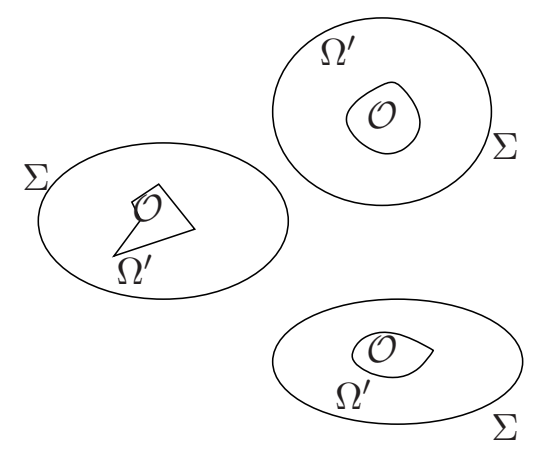

Figure 5.1: Bounded domain $\Omega^{\prime}$

where $\Sigma=\partial \Omega^{\prime} \backslash \partial \mathcal{O}$ and $\Theta_{\Sigma}=(\partial / \partial n+\beta), \beta$ is an arbitrary complex parameter.

It is clear that if $\varphi_{D}^{(1)}$ is a solution of (4.1) then $\varphi^{\prime}=\varphi_{D_{/ \Omega^{\prime}}}^{(1)}$ is solution of problem (5.1). Similarly, provided $\operatorname{Im}(\beta) \neq 0$, every solution $\varphi^{\prime}$ of (5.1) can be uniquely extended to a solution $\varphi_{D}^{(1)}$ of (4.1) by the integral representation formula on $\partial \mathcal{O}$ :

$$
\forall x \in \Omega_{\mathcal{O}}, \varphi_{D}^{(1)}(x)=\int_{\partial \mathcal{O}}\left\{G_{0}(x, y) \frac{\partial \varphi^{\prime}}{\partial n}(y)-\frac{\partial G_{0}}{\partial n_{y}}(x, y) \varphi^{\prime}(y)\right\} d \sigma(y) .
$$

The variational formulation of the problem (5.1) is:

$$
\left\{\begin{array}{l}
\text { Find } \varphi^{\prime} \in H^{1}\left(\Omega^{\prime}\right) \text { such that } \forall \psi \in H^{1}\left(\Omega^{\prime}\right), \text { we have } \\
\int_{\Omega^{\prime}} \nabla \varphi^{\prime} \cdot \overline{\nabla \psi}-k^{2} \int_{\Omega^{\prime}} \varphi^{\prime} \bar{\psi}-\beta \int_{\Sigma} \varphi^{\prime} \bar{\psi}+ \\
\int_{\Sigma}^{\bar{\psi}(x)} \int_{\partial \mathcal{O}} \varphi^{\prime}(y)\left(\frac{\partial}{\partial n_{x}}+\beta\right) \frac{\partial G_{0}}{\partial n_{y}}(x, y) d \sigma(y) d \sigma(x)= \\
-\int_{\partial \mathcal{O}} h \bar{\psi}+\int_{\Sigma} \overline{\psi(x)} \int_{\partial \mathcal{O}} h(y)\left(\frac{\partial}{\partial n_{x}}+\beta\right) G_{0}(x, y) d \sigma(y) d \sigma(x) .
\end{array}\right.
$$

Finally, we discretize problem (5.3) to obtain a linear system that we solve numerically.

\subsection{Numerical results}

All the numerical results are obtained by the code MELINA [11]. We consider an oblong mirror of width 8 and height 1 and two half-disk obstacles of 
diameters 4 and 2 (see figure 5.2). We denote by $D$ the distance between the mirror and the obstacles. The distance between the scatterers is $\delta=2$. We investigate the two cases: $D=3$ and $D=8$. We consider here a Neumann condition on the boundary of the mirror $\partial M(\mu=0$, that is, the case of a large acoustic impedance of the transducers).

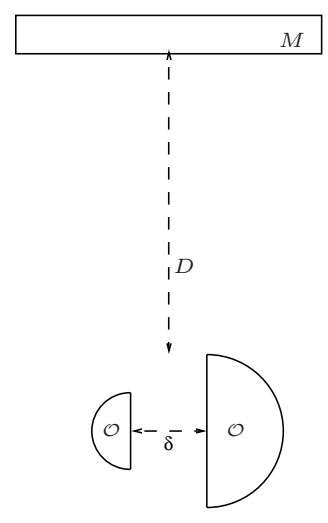

Figure 5.2: Geometry of the problem

Figure 5.3 (respectively, 5.4) shows the amplitude of the total field corresponding to the emission of the first (respectively, second) eigenvector associated to $\lambda_{1}=0.0499$ if $D=8$ and $\lambda_{1}=0.2211$ if $D=3$ (respectively, $\lambda_{2}=0.0191$ if $D=8$ and $\lambda_{2}=0.0534$ if $D=3$ ) in the case of the first model presented in section 2 and where $k=3.14$ (the wavelength $l_{w}=2 \pi / k=2$ is then equal to the distance between the obstacles $\delta$ ). We observe that the wave is focused on the biggest obstacle (respectively, the smallest). When emitting the third eigenvector associated to $\lambda_{3}=0.0002$ if $D=8$ and $\lambda_{3}=0.0085$ if $D=3$, we see that there is no focusing on the obstacles (see figure 5.5). These results essentially show that selective focusing, which is known to occur for small and distant enough scatterers [8], is achieved although the size of the obstacles, the distance between them and the wavelength are of the same order.

Figure 5.6 shows the first four eigenvalues of the time reversal operator $T$ according to the wave number $k$ in the case of the first model presented in section 2 where the interactions between the mirror and the obstacles are taken into account and where the distance $D$ between them is respectively 

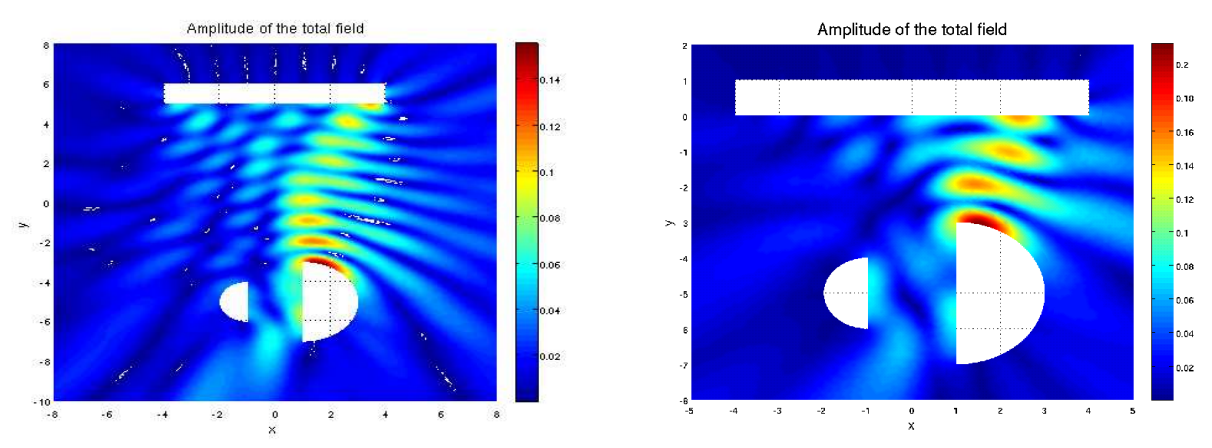

Figure 5.3: Emission of the first eigenvector $(D=8$ in the left, $D=3$ in the right).
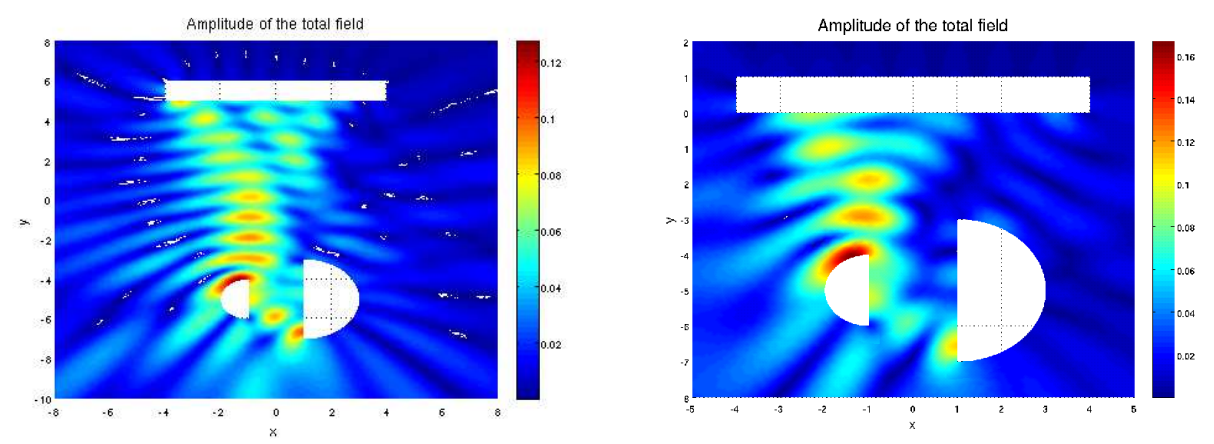

Figure 5.4: Emission of the second eigenvector $(D=8$ in the left, $D=3$ in the right).

3 and 8. Figure 5.7 shows the same results in the case of the second model, where these interactions are approximated by only the first reflections.

According to figures 5.6 and [5.7, there is only one significant eigenvalue $\lambda_{1}$ at low frequencies. This can be explained by the fact that the wavelength $l_{w}$ is wider than the distance $\delta$ between the two obstacles, so the mirror see them as only one. Figure 5.9 (respectively, 5.10) shows the amplitude of the total field corresponding to the emission of the first (respectively, second) eigenvector associated to $\lambda_{1}=0.6994$ if $D=8$ and $\lambda_{1}=3.2717$ if $D=3$ (respectively, $\lambda_{2}=0.0006$ if $D=8$ and $\lambda_{2}=0.0265$ if $D=3$ ) in the case of the first model presented in section 2 and where $k=0.325$. We observe in figure 5.9 that the mirror can not distinguish between the obstacles and sees them as only one. 

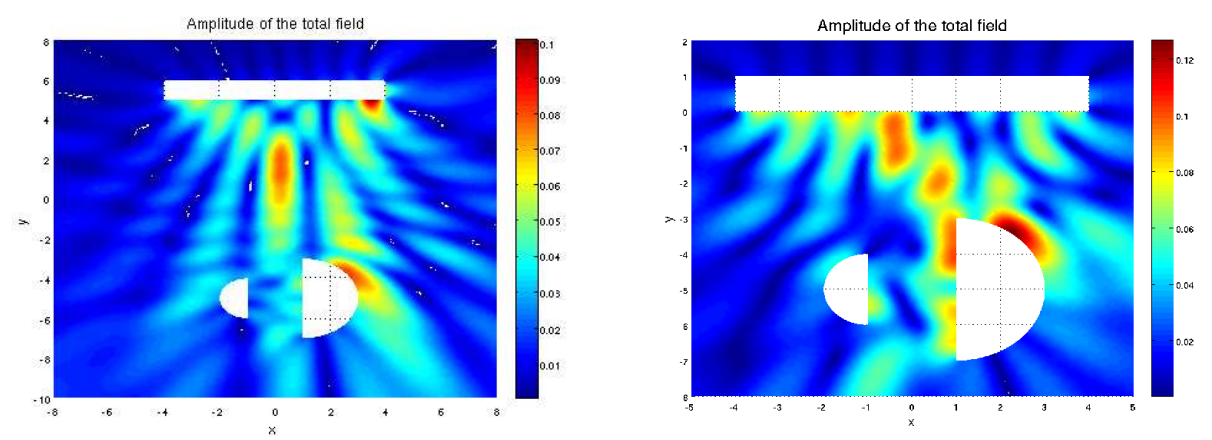

Figure 5.5: Emission of the third eigenvector $(D=8$ in the left, $D=3$ in the right).
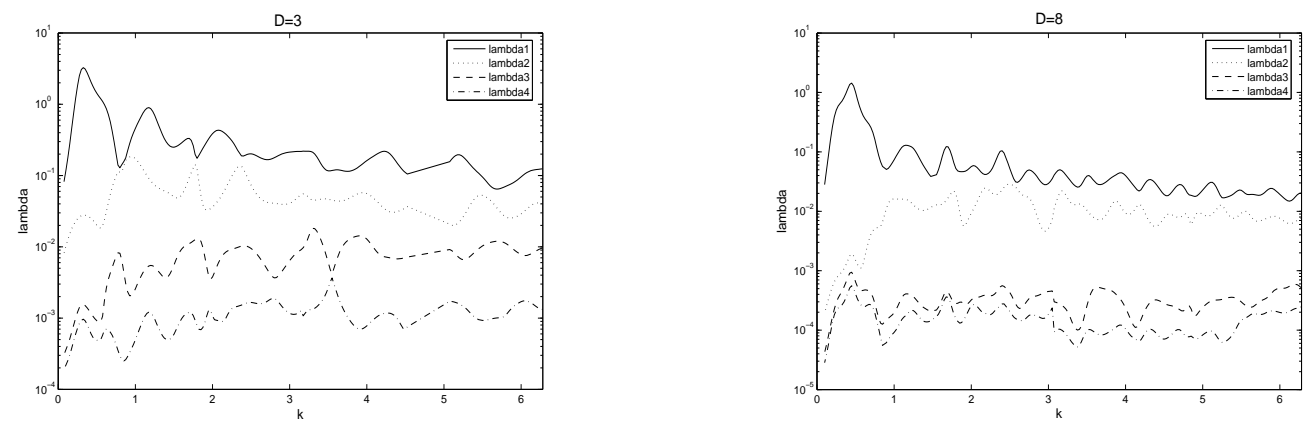

Figure 5.6: First model: 4 largest eigenvalues of $T$ according to $k$.

When $k$ increases, figures 5.6 and 5.7 show that there are two significant eigenvalues in the case where $D=8$. However, they show that the third eigenvalue is not so negligible in comparison with the second one when $D=3$. The fact that the gap between the two largest eigenvalues and the following ones is increased when the TRM moves away $(D=8)$ is related to the angular aperture under which the TRM is seen from the obstacles.

At medium and high frequencies, we note the presence of important oscillations of the first two eigenvalues of $T$ when the interactions between the mirror and the obstacles are taken into account (figure 5.6) contrarily to the case where these interactions are approximated (figure 5.7).

To understand this, we show in figure 5.8 the first two eigenvalues of the two models where respectively $D=3$ and $D=8$. We remark that each eigenvalue (first or second) corresponding to the model which takes into account 

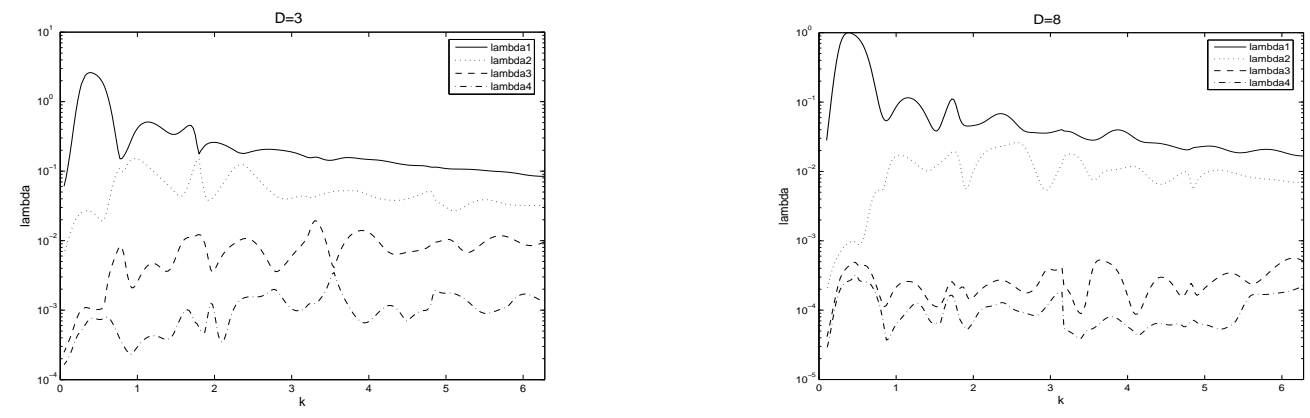

Figure 5.7: Second model: 4 largest eigenvalues of $T$ according to $k$.
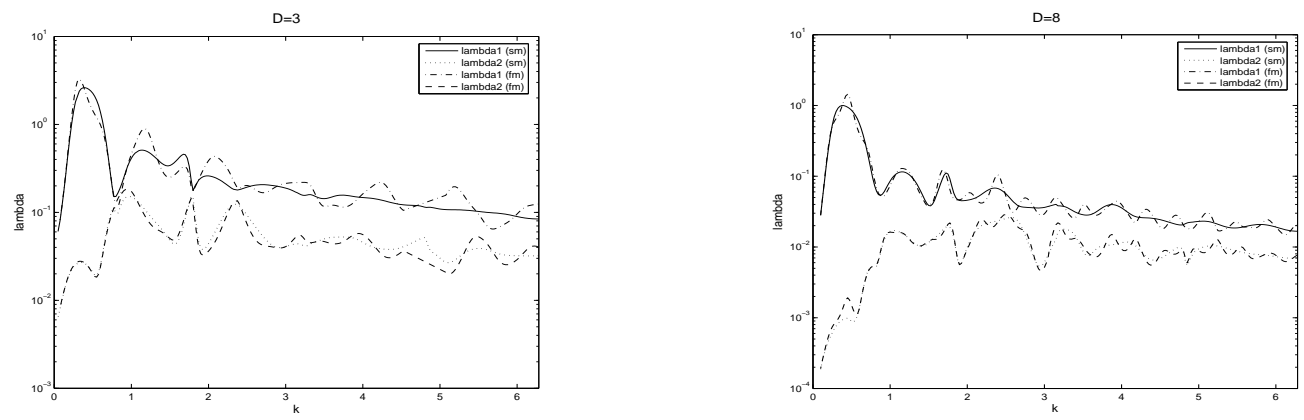

Figure 5.8: The first two eigenvalues for the 2 models. 

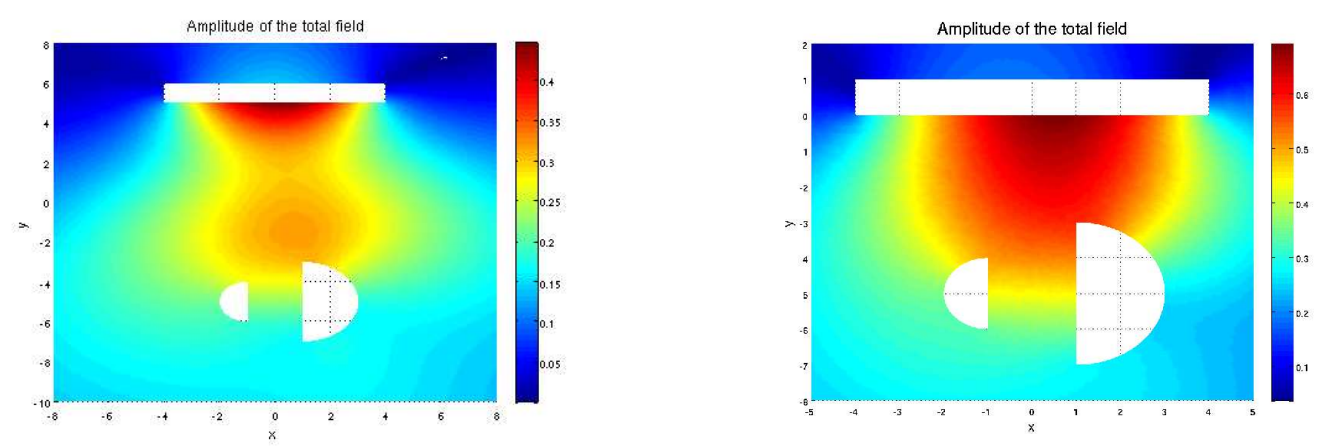

Figure 5.9: Emission of the first eigenvector $(D=8$ in the left, $D=3$ in the right).
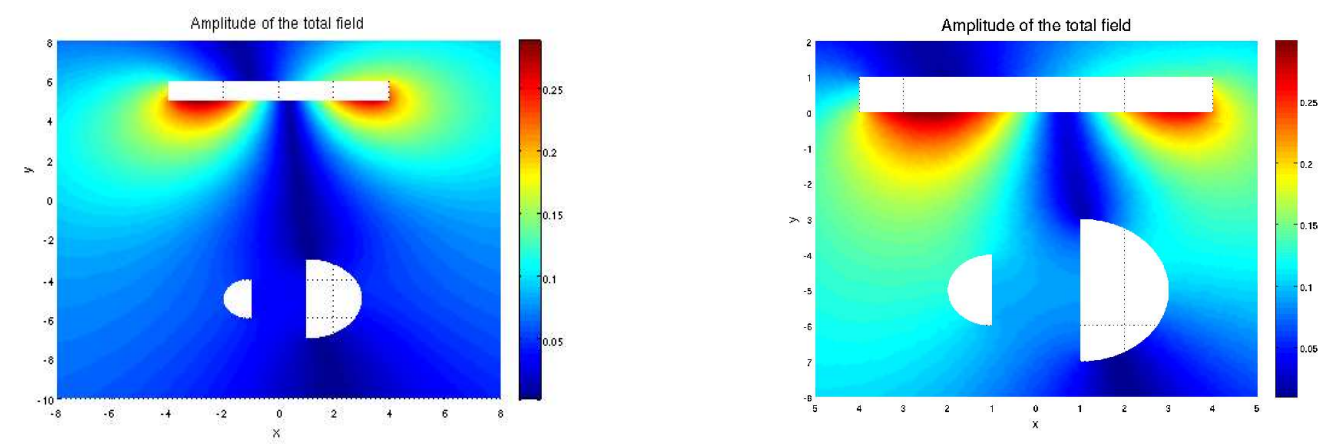

Figure 5.10: Emission of the second eigenvector $(D=8$ in the left, $D=3$ in the right).

the interactions between the mirror and the obstacles oscillates around the corresponding eigenvalue where we consider only the first reflections between them. This can be explained by the fact that the interactions between the mirror and the obstacles can be constructive or destructive according to the distance between the mirror and the obstacles and to the shape of the obstacles. We note the dependence of the period of oscillations on the distance between the mirror and the obstacles: $\Delta k \simeq \pi / D$.

We also remark that the interactions between the mirror and the obstacles are constructive for the wave numbers $k_{n}=n \pi / D, n \in \mathbb{N}^{*}$ and destructive for $k_{n}=((n-1 / 2) \pi) / D, n \in \mathbb{N}^{*}$. This can be understood by the fact that the wave numbers $k_{n}=n \pi / D, n \in \mathbb{N}^{*}$ represent the eigenvalues of the operator $-\Delta$ in the one dimensional domain $[0, D]$ and the corresponding eigenfunctions when considering Neumann conditions on the boundaries are 


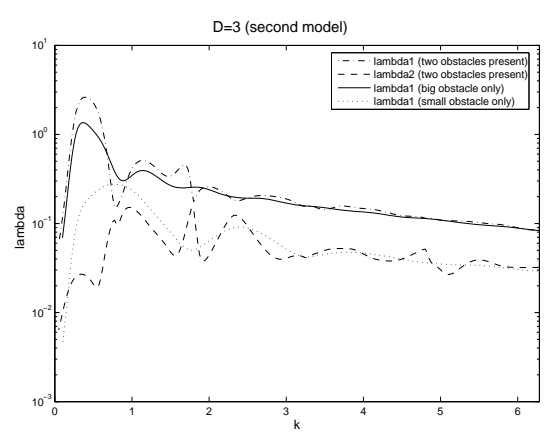

Figure 5.11: Analysis of the interactions between obstacles

$\varphi_{n}(y)=\cos \left(k_{n} y\right), y \in[0, D]$.

To study the interactions between the obstacles, we show now in figure 5.11 the first and second eigenvalues of $T$ for the approximate model and for $D=3$, together with the first eigenvalue of $T$ for the same model but with a new geometrical configuration in which only the biggest or the smallest obstacle is present. We observe that, at medium frequencies, there is a perfect coincidence between the first eigenvalue of $T$ corresponding to the case where the two obstacles are present and the one where there is only the biggest obstacle, which explains that the interactions due to the smallest obstacle are negligible. Meanwhile, the second eigenvalue of $T$ corresponding to the case where the two obstacles are present oscillates smoothly around the first eigenvalue where there is only the smallest obstacle, which proves that the interactions due to the biggest obstacle are important.

We remark that the two greatest eigenvalues are very close for $D=3$ and $k=0.875, \lambda_{1}=0.1760$ and $\lambda_{2}=0.1637$ (see figure 5.6). To understand this phenomenon, we show in figure 5.12 (respectively, 5.13) the amplitude of the total field corresponding to the emission of the first (respectively, second) eigenvector associated to $\lambda_{1}=0.0507$ if $D=8$ and $\lambda_{1}=0.1760$ if $D=3$ (respectively, $\lambda_{2}=0.0092$ if $D=8$ and $\lambda_{2}=0.1637$ if $D=3$ ) for the first model presented in section 2 and for $k=0.875$ (the wavelength $l_{w}=2 \pi / k=2$ is then equal to 7.18). It is clear that the TRM distinguishes the two targets which are separated by less than a third of a wavelength when the TRM is located less than half a wavelength away from them. This effect results from the proximity of the TRM $(D=3)$, not because it is a TRM, but because 
it acts as a probe in the near-field (see also near-field microscocy in Optics [?]). However, we observe that the wave is not concentrated only on the obstacles. We verify by figures 5.12 and 5.13 that this effect is not observed when the TRM is moved away $(D=8)$. When emitting the third eigenvector associated to $\lambda_{3}=0.000016$ if $D=8$ and $\lambda_{3}=0.0029$ if $D=3$, we see in figure 5.14) that the obstacles are not illuminated.

\section{References}

[1] J.P. Aubin, Analyse Fonctionnelle Appliquée (Tome II), Presses Universitaires de France, 1987.

[2] M. Balabane, Boundary decomposition for Helmholtz and Maxwell equations 1: disjoint sub-scatterers, Asymptotic Analysis, 38 (2004), pp. 1-10.

[3] M. Balabane And V. Tirel, Décomposition de domaine pour un calcul hybride de l'équation de Helmholtz, C. R. Acad. Sci. Paris, 324 (1997), pp. 281-286.

[4] L. Borcea, G. Papanicolaou, C. Tsogka and J. Berryman, Imaging and time reversal in random media, Inverse Problems, 18 (2002), pp. 1247-1279.

[5] D. Colton And R. KRess, Inverse Acoustic and Electromagnetic Scattering Theory, Springer-Verlag, Berlin, 1993.

[6] E. Domany and O. Entin-Wohlman, Application of multiple scattering theory to subsurface defects, J. Appl. Phys., 56 (1984), pp. 137142.

[7] R. Dautray And J.L. Lions, Analyse Mathématique et Calcul Numérique pour les Sciences et les Techniques (Tome I), Masson, Paris, 1985.

[8] C. Hazard, and K. Ramdani, Selective acoustic focusing using timeharmonic reversal mirrors, SIAM J. App. Math., 64 (2004), pp. 10571076. 
[9] A. JAmi, AND M. LenOIR, A variational formulation for exterior problems in linear hydrodynamics, Comput. Methods Appl. Mech. Engrg., 16 (1978), pp. 341-359.

[10] J.F. Lingevitch, H.C. Song and W.A. Kuperman, Time reversed reverberation focusing in a waveguide, J. Acoust. Soc. Am., 111 (2002).

[11] D. Martin, Mélina: http://anum-maths.univ-rennes1.fr/melina/.

[12] P.A. Martin, Multiple Scattering: Interaction of Time-Harmonic Waves with $N$ Obstacles, Cambridge, 2006.

[13] T.D. Mast, A.I. Nachman And R.C. WAAG, Focusing and imaging using eigenfunctions of the scattering operator, J. Acoust. Soc. Am., 102 (1997), pp. 715-725.

[14] G. Micolau And M. Saillard, D.O.R.T method as applied to electromagnetic subsurface sensing, Radio Science, 38 (2003).

[15] N. Mordant, C. PRADA, AND M. Fink, Highly resolved detection and selective focusing in a waveguide using the D.O.R.T method, J. Acoust. Soc. Am., 105 (1999), pp. 2634-2642.

[16] A.D. PIERCE, Acoustics, an introduction to its physical principle and applications, McGraw-Hill, New York, 1981.

[17] C. PRADA AND M. FINK, Eigenmodes of the time reversal operator: a solution to selective focusing in multiple-target media, Wave motion, 20 (1994), pp. 2067-2076.

[18] C. Prada, E. Kerbrat, D. Cassereau and M. Fink, Time reversal techniques in ultrasonic nondestructive testing of scattering media, Inv. Prob., 18 (2002), pp. 1761-1773.

[19] C. Prada, S. Manneville, D. Spoliansky and M. Fink, Decomposition of the time reversal operator: Detection and selective focusing on two scatterers, J. Acoust. Soc. Am., 99 (1996), pp. 2067-2076.

[20] H.A. SCHENCK, Helmholtz integral formulation of the sonar equations, J. Acoust. Soc. Am., 79 (1986), pp. 1423-1433. 
[21] G.T. Schuster, A hybrid BIE+Born series modeling scheme: generalized Born series, J. Acoust. Soc. Am., 77 (1985), pp. 865-879.

[22] H. Tortel, G. Micolau, and M. Saillard, Decomposition of the time reversal operator for electromagnetic scattering, J. Electromag. Waves and App., 13 (1999), pp. 687-719. 

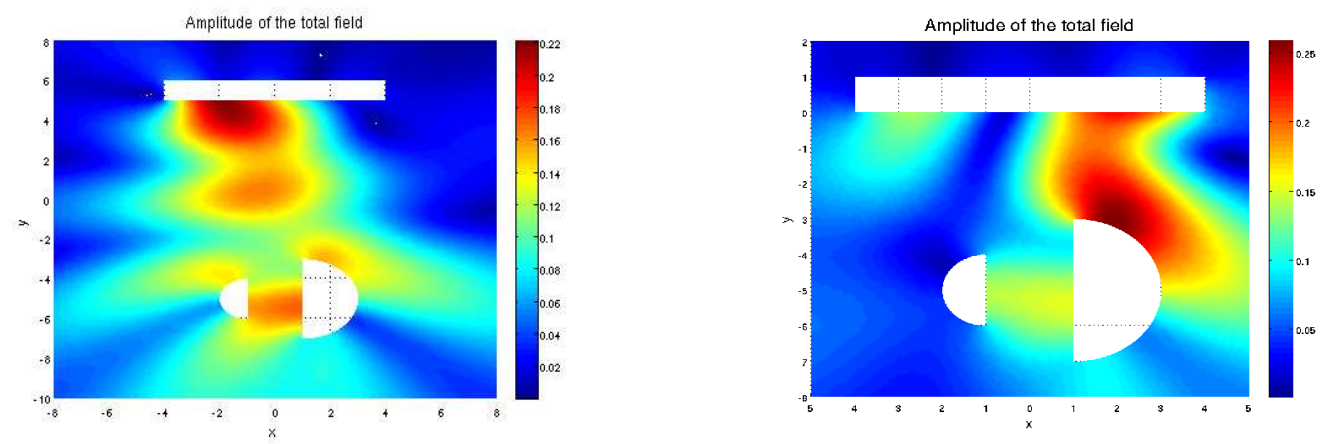

Figure 5.12: Emission of the first eigenvector $(D=8$ in the left, $D=3$ in the right).
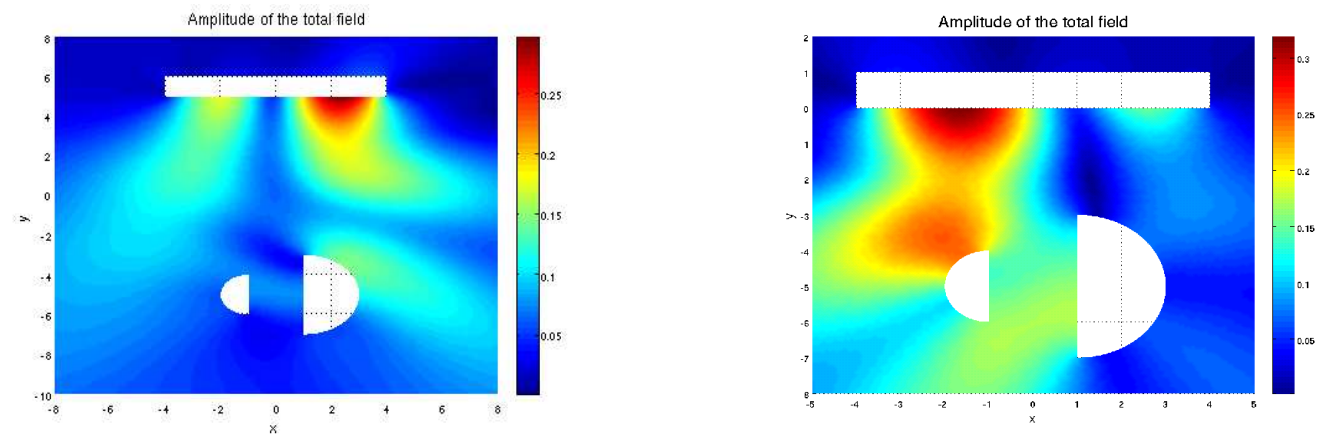

Figure 5.13: Emission of the second eigenvector $(D=8$ in the left, $D=3$ in the right).
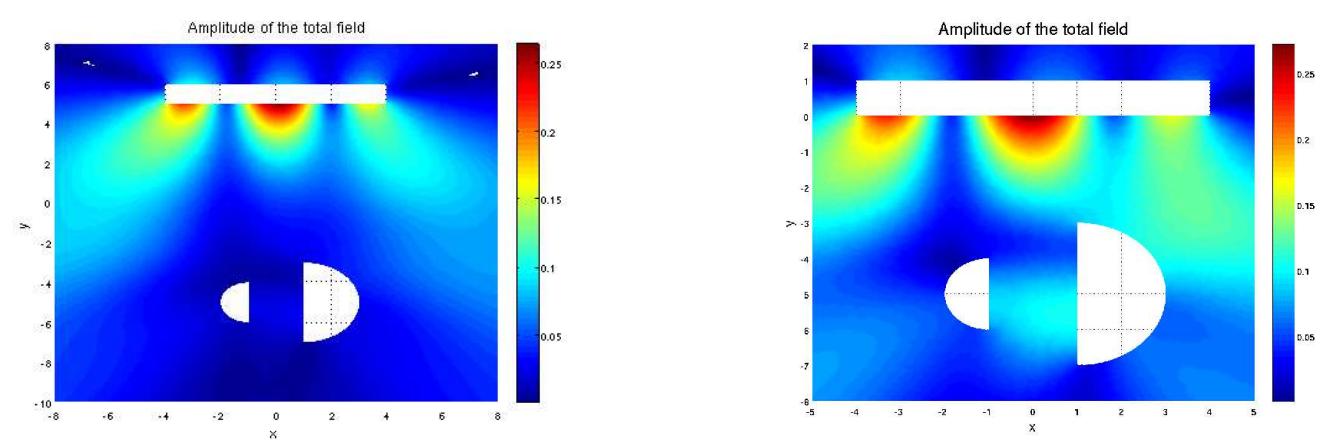

Figure 5.14: Emission of the third eigenvector $(D=8$ in the left, $D=3$ in the right). 